\title{
Implementation of a direct install 3-pole type EM transducer in round window niche for implantable middle ear hearing aids
}

\author{
Dong Ho Shin ${ }^{\mathrm{a}}$, Hyung-Gyu Lim ${ }^{\mathrm{a}}$, Eui Sung Jung ${ }^{\mathrm{a}, \mathrm{c}}$, Qun Wei ${ }^{\mathrm{a}}$, Ki Woong Seong ${ }^{\mathrm{b}}$, Jyung \\ Hyun Lee ${ }^{\mathrm{b}}$, Seung-ha Lee ${ }^{\mathrm{d}}$ and Jin Ho Cho ${ }^{\mathrm{a}, \mathrm{c}, *}$ \\ ${ }^{a}$ Graduate School of Electronics Engineering, Kyungpook National University, 80 Daehakro, Buk-Gu, \\ Daegu 702-701, S. Korea \\ ${ }^{b}$ Department of Biomedical Engineering, Kyungpook National University Hospital, 130 Dongdeok-ro, \\ Jung-Gu, Daegu 700-721, S. Korea \\ ${ }^{c}$ School of Electronics Engineering, College of IT Engineering, Kyungpook National University, \\ Sangyuk-dong, Buk-Gu, Daegu 702-701, S. Korea \\ ${ }^{d}$ Department of Biomedical Engineering, College of Medicine, Dankook University, 119 Dandae-ro, \\ Dongnam-Gu, Cheonan-si, Chungnam 330-714, S. Korea
}

\begin{abstract}
Since the 1980's, various types of implantable hearing aids using unique means for delivering acoustic power to the inner ear have been developed. Recently, implantable hearing aids that stimulate the round window by the middle ear transducer have received great attention because it reduces loading effect at the ossicular chain. In this study, we have implemented a direct install 3-pole type EM transducer in round window niche for implantable middle ear hearing aid. The 3pole type EM transducer consists of two permanent magnets and three coils and exhibit structural features that minimize leakage flux, thereby permitting high efficiency and low magnetic field interference. The stapes velocity was measured using a laser Doppler vibrometer in response to the round window stimulation from the transducer. To verify the usefulness of the 3-pole type EM transducer, we compared the stapes vibration characteristics produced by the transducer and those from a sound source. The magnitude of stapes velocity due to the round window stimulation at $1 \mathrm{~mA}_{\mathrm{rms}}$ was equivalent to that of stapes velocity at $94 \mathrm{~dB}$ SPL sound stimulation. Thus, the evaluation study shows that the 3-pole type EM transducer is suitable for implantable hearing devices.
\end{abstract}

Keywords: 3-pole type EM transducer, round window, stapes velocity, implantable hearing aids

\section{Introduction}

Hearing aids with various types of acoustic power transmission mechanisms to the inner ear have been developed. A typical example is ossicular chain driving-type implantable middle ear hearing devices (IMEHDs) [1-4]. The ossicular chain driving-type IMEHDs consist of a transducer that gene-

\footnotetext{
${ }^{*}$ Corresponding author: Jin Ho Cho, Graduate School of Electronics Engineering, Kyungpook National University, 80 Daehakro, Buk-Gu, Daegu 702-701, S. Korea. Tel.: +82-53-427-5538; Fax: +82-53-427-5539; E-mail: jhcho@ee.knu.ac.kr.
}

0959-2989/14/\$27.50 @ 2014 - IOS Press and the authors. 
rates mechanical vibration and an attached clip for the long process of the incus. To maximize transmission efficiency of the vibration, strong coupling between the long process of the incus and the transducer is needed. However, if the clip is strongly attached to the ossicles, it may cause necrosis of the ossicles [5,6]. Furthermore, when the ossicles do not exist or the transfer characteristics of them differ greatly from the normal case, the ossicular chain driving-type transducer cannot be used.

To solve these issues, round window driving-type implantable hearing devices have been used [7-9]. The round window driving method involves the transmission of the vibration signal to the round window of the inner ear, such that stimulation to an oval window occurs in the opposite direction. In the round window method, there are two ways in which vibrational energy is delivered. In the first method, the transducer is mounted on the exterior wall of the tympanic cavity and stimulates the round window through the connecting material (silicone rod, wire rod, etc.). Alternatively, a miniaturized transducer is installed on the round window niche and stimulates the round window membrane directly [10-13].

The advantage of mounting the transducer on the exterior wall of the tympanic cavity is such that high power can be obtained. However, it is difficult to implement the support structure for fixing the transducer inside the middle ear cavity. In addition, design of connecting material between the transducer and the round window is tricky because it must avoid the facial nerve. When the transducer is mounted in the round window niche, due to space limitations, the size of the transducer must be much smaller than the exterior type. As a result, the vibrational force of the transducer is also reduced. However, this method has the advantages of easy mounting and high coupling efficiency between the transducer and the round window without any connecting material.

In this study, we have implemented a 3-pole type electromagnetic (EM) transducer that can be implanted directly in the round window niche. The vibrational characteristics of the proposed transducer were measured. To verify the usefulness of the round window driving 3-pole type EM transducer, we compared the stapes vibrational characteristics obtained by the proposed transducer and sound pressure, respectively.

\section{Materials and methods}

\subsection{Temporal bone specimens}

Temporal bone specimens from three human ears were used in this study. TB1 were extracted from a 79-year-old Caucasoid male, TB2 came from an 86-year-old Caucasoid male, and TB3 were extracted from an 80-year-old Caucasoid male. All specimens were obtained from the Anatomy Gifts Registry in 2013 and were frozen after extraction. The human temporal bone specimens were prepared by performing a posterior tympanotomy. Then, the facial nerve and the bony protrusion of the round window niche were removed to allow the membrane of the transducer to be nearly horizontal with the round window membrane. The external ear canal was removed and replaced with an artificial ear canal. In addition, reflective beads for reflecting the laser were placed on the stapes footplate.

\subsection{Round window driving 3-pole type EM transducer}

The structure of the 3-pole type EM transducer is shown in Figure 1(a). The transducer has a diameter of $1.75 \mathrm{~mm}$ and a length of $3.0 \mathrm{~mm}$. There are three coils; one of the coils is placed in the forward direction, and the other coils are placed in the opposite direction. Thus, this structure can generate force in one direction. Two magnets (D: $1.0 \mathrm{~mm}, \mathrm{~L}: 1.0 \mathrm{~mm}$ ) in the coil are glued back to back with 
the same poles facing. Consequently, any forces generated due to an external magnetic field are canceled out. To determine the optimal ratio of coil length, finite element analysis was performed. The result is shown in Figure 1(b). When the ratio of coils was 0.9:1.2:0.9, maximum Lorentz force was generated [14].

\subsection{Experimental procedure}

First, an acoustic signal of $94 \mathrm{~dB}(1 \mathrm{~Pa})$ was injected into the ear canal, and the stapes head response was measured using a laser Doppler vibrometer (LDV). The angle of incidence of the laser in the LDV did not exceed $60^{\circ}$ from normal to the stapes head. The acoustic signal was calibrated using a probe microphone (ER-7C). The tip of the probe microphone was positioned at a distance of 1 to $2 \mathrm{~mm}$ from the center of the tympanic membrane. The measured results are shown in Figure 2 and satisfy ASTMF2504 [15].

Second, the 3-pole type EM transducer contacted the round window membrane, and vibrations in either the stapes footplate or the stapes head was measured with LDV. In the case of the round window

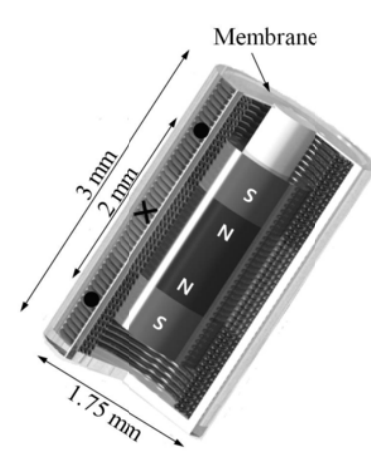

(a)

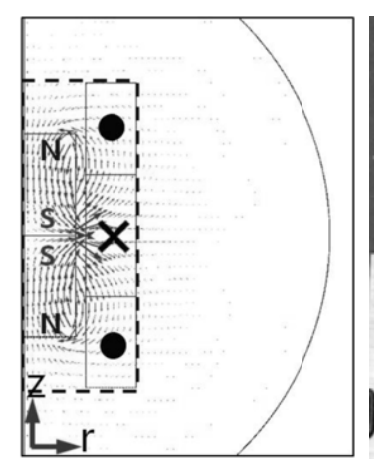

(b)

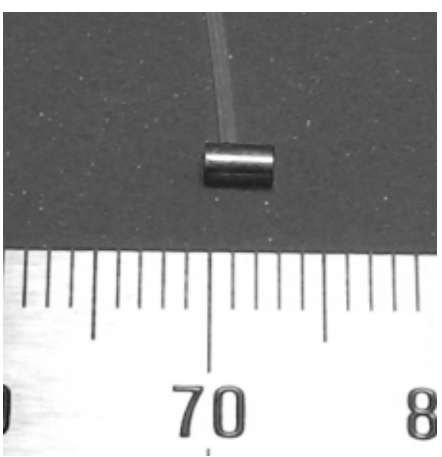

(c)

Fig. 1. The 3-pole type EM transducer: (a) the structure, (b) flux line due to the permanent magnet, and (c) photo of the implemented 3-pole EM transducer.

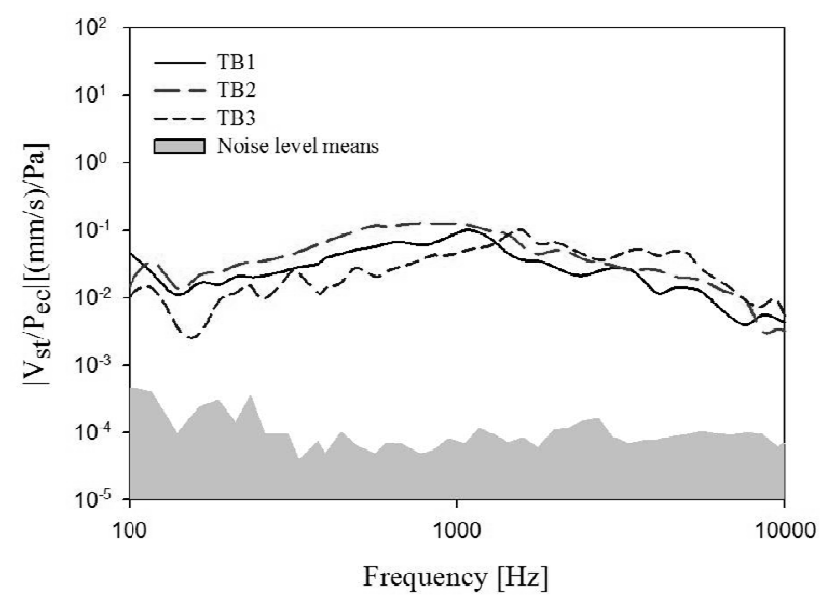

Fig. 2. Stapes vibration velocity of temporal bones at $94 \mathrm{~dB}$ SPL. 
stimulation, the source of vibration for driving the transducer was $1 \mathrm{~mA}_{\mathrm{rms}}$ between $0.1 \mathrm{kHz}$ and 10 $\mathrm{kHz}$. The driving current used in implantable hearing aid transducers is $1 \mathrm{~mA}_{\mathrm{rms}}[16,17]$. Therefore, the driving current of the 3-pole type EM transducer was selected as $1 \mathrm{~mA}_{\mathrm{rms}}$. Self-vibration characteristics of the 3-pole type EM transducer are shown in Figure 3. The transducer showed a resonance frequency of $3 \mathrm{kHz}$.

\subsection{Measurement system and 3-pole type EM transducer attached to round window}

According to previous studies, the reverse stimulation with a transducer produced differential pressure comparable to forward stimulation with sound introduced into the ear canal. Also, the reverse stimulation produced stapes vibration comparable to forward stimulation. Therefore, we utilized the stapes measurement methods from previous studies $[8,15]$. The preparation of the temporal bone specimens and the experimental setup are shown in Figure 4. Vibration measurements were performed using a data acquisition system (National Instruments Co., USA, NIPXI-1042) and LDV (Polytec GmbH, Germany, OFV-551 \& OFV-5000). This system acquired data from the LDV at each frequency using a fast Fourier transformation. The reflective beads targeted by the laser were positioned through a microscope [11].

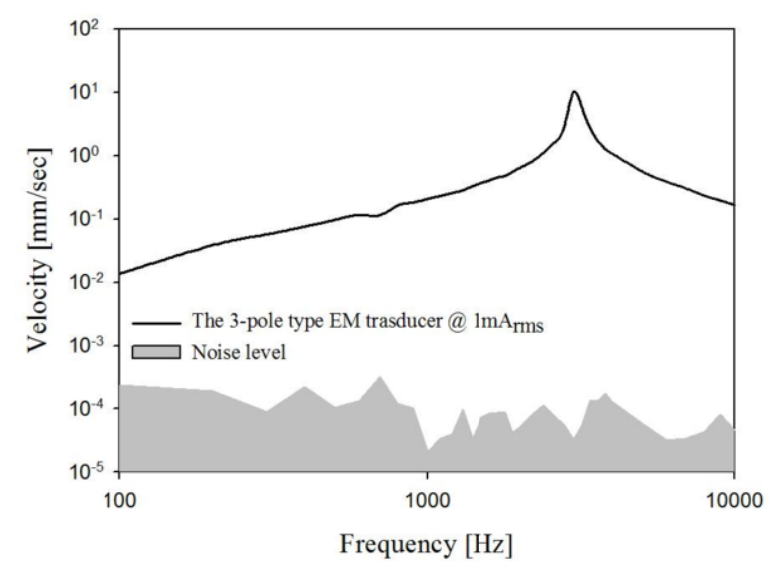

Fig. 3. Self-vibration characteristics of the 3-pole type EM transducer.
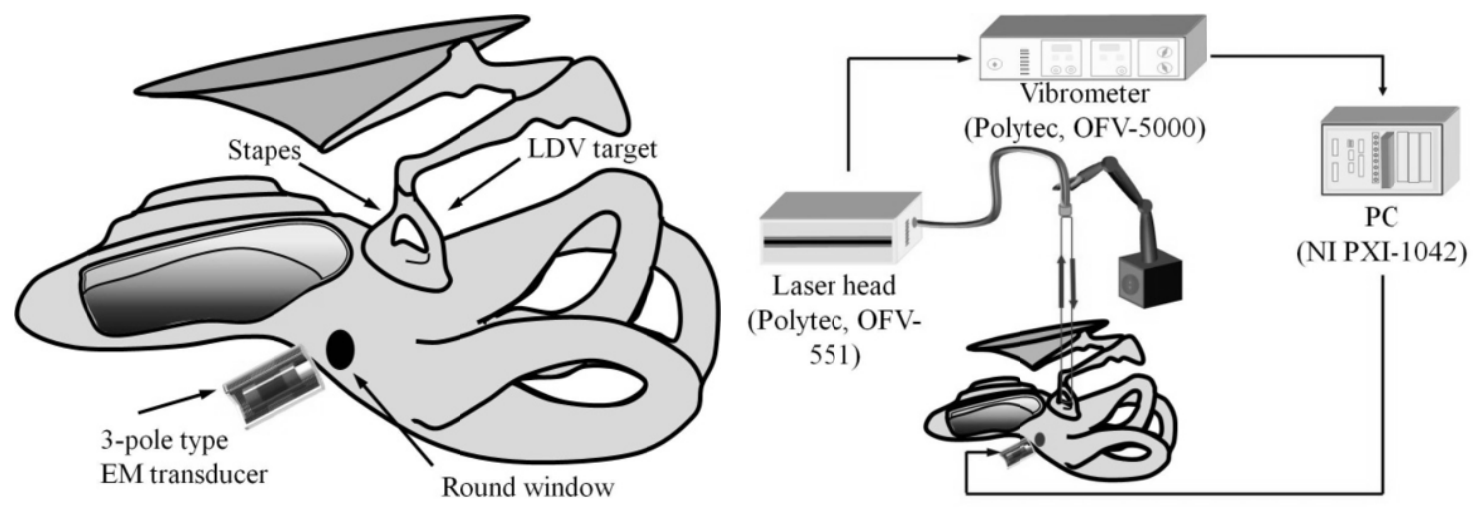

Fig. 4. Experiments for measuring the temporal bone stapes velocity using the LDV [8]. 

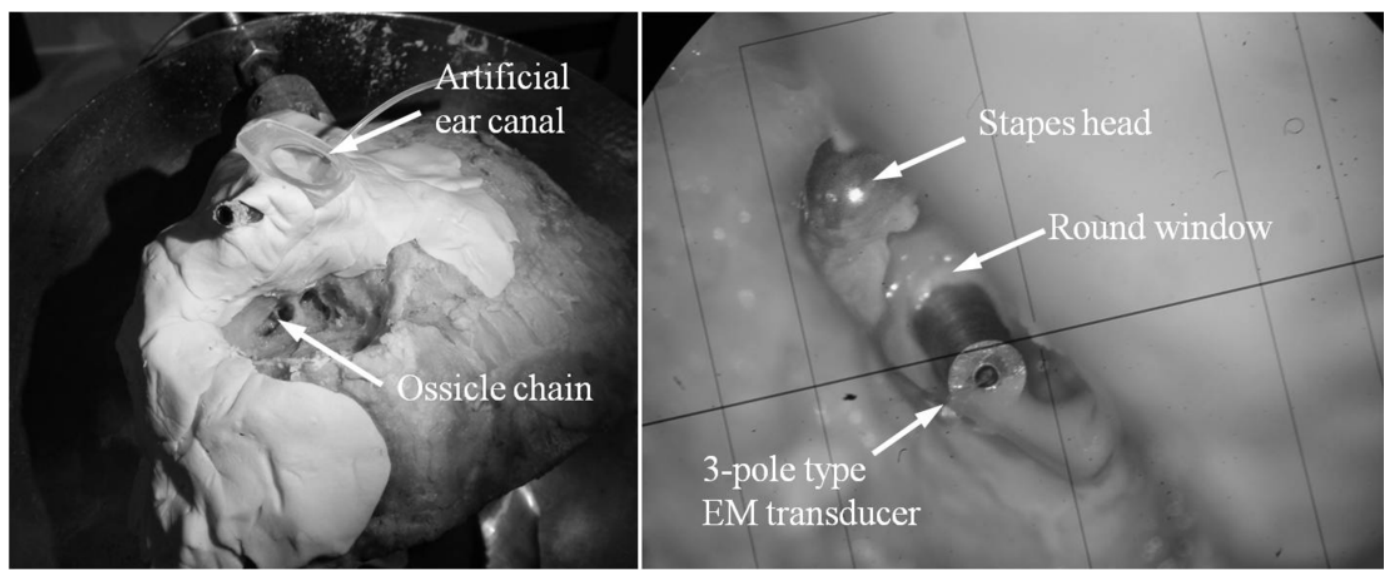

Fig. 5. The 3-pole type EM transducer in contact with round window membrane.

The 3-pole type EM transducer installed on the round window is as shown in Figure 5. By wrapping the transducer using a slight patch of fascia and then inserting it inside the round window niche, the installation is easily performed. The measurements are then repeated using the same 3-pole type EM transducer. The three human temporal bone specimens were attached to the round window using the same method.

\section{Result}

The round window stimulation was driven with the 3-pole type EM transducer, and the stapes velocity was measured simultaneously. The frequency characteristics of the stapes velocity for the three temporal bone specimens are shown in Figure 6. The stapes characteristics of temporal bones using the round window were similar to the stapes velocity requirements given in ASTM-2504. The noise level did not affect the measurements.

The results for all temporal bones, i.e., TB1 TB3, showed that the magnitude of the round window stimulation and tympanic membrane stimulation were similar. However, the magnitude of tympanic membrane stimulation was higher than that of the round window stimulation at low frequency. These results can be attributed to two reasons. First, the inherent characteristic of the implemented transducer has a slightly lower gain below the $1 \mathrm{kHz}$ region. Second, there is suboptimal coupling between the 3pole type EM transducer and the round window membrane especially in the case of TB 3 . Thus, for practical applications, at $1 \mathrm{~mA}_{\mathrm{rms}}$ driving current, the vibrational intensity was slightly insufficient for low frequency ranges. Therefore, more driving current are needed to produce the equivalent acoustic level with $94 \mathrm{~dB}$ SPL.

When the round window and the transducer are not optimally coupled, the vibration is not transmitted efficiently inside the cochlear. Also, if the transducer is strongly attached to the RW, it was caused the membrane mass increase of transducer. In this case, the movement of the resonance frequency occurs. Therefore, the resonance frequency is generated different each other position as shown in Figure 6.

Next, the results of the round window stimulation using the 3-pole type EM transducer were compared with those from similar studies. Although we used the same experimental approach as other stu- 
dies, however, the transducer of stimulation round window are using other types of structure. The characteristics of each transducer are different, but the characteristics at the low-frequency region are very

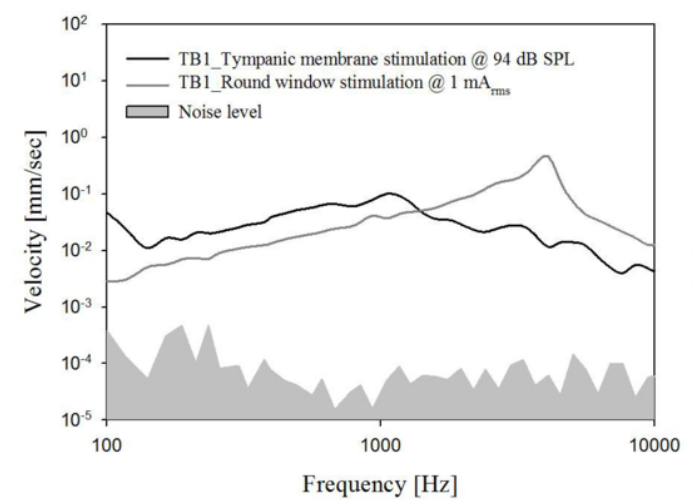

(a)

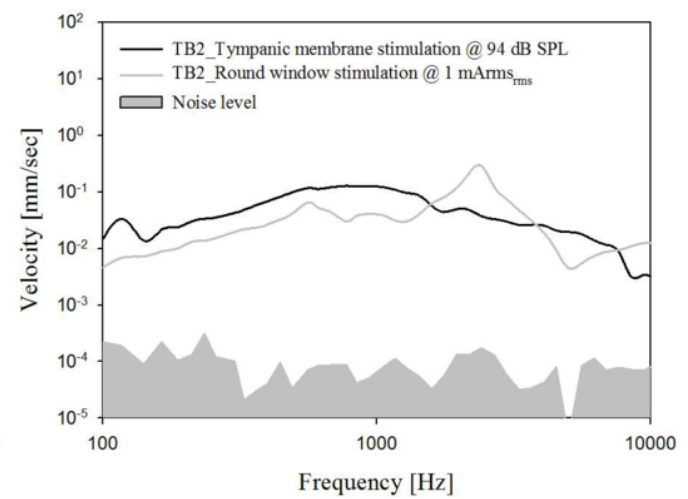

(b)

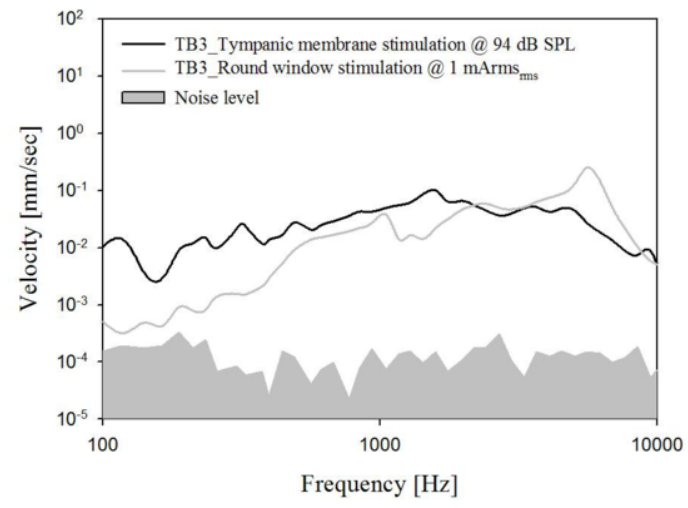

(c)

Fig. 6. Stapes vibration characteristics generated by the 3-pole type EM transducer.

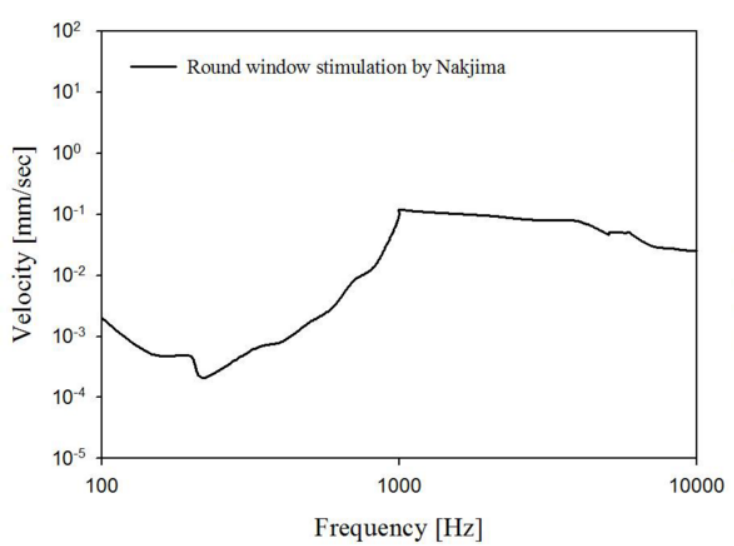

(a)

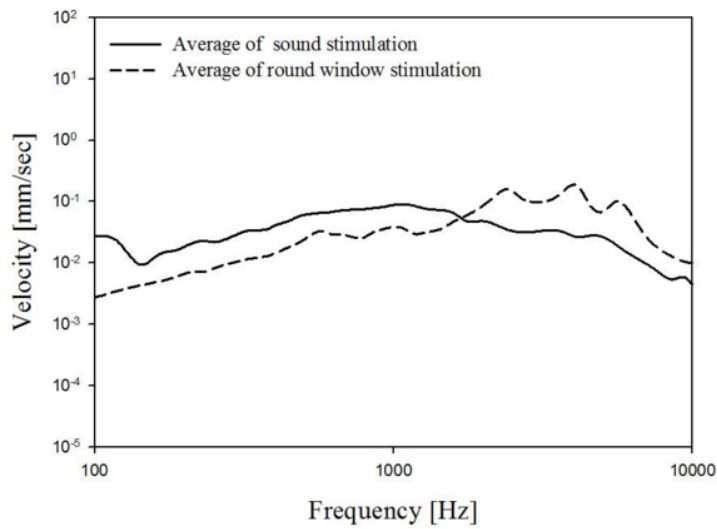

(b)

Fig. 7. (a) Stapes velocity magnitude response due to FMT stimulation with $100 \mathrm{mVp}$-p drive level [8], (b) Stapes velocity magnitude response due to 3-pole type EM transducer stimulation with $1 \mathrm{~mA}_{\mathrm{rms}}$ drive level $(\mathrm{n}=3)$. 
similar. Figure 7(a) shows the data acquired by Nakajima [8]. A comparison of Figure 7 shows that round window stimulation with the 3-pole type EM transducer performed better at low frequencies $(0.1 \mathrm{kHz}$ to $1 \mathrm{kHz})$ than that of floating mass transducer (FMT).

\section{Conclusion}

In this paper, we show that the implemented 3-pole type EM transducer for implantable hearing system can be easily installed on the round window membrane. The 3-pole type EM transducer showed high performance at low frequency than conventional models. Three temporal bone specimens were used in this study. For all the temporal bones tested, the magnitude of stapes velocity by the round window stimulation was comparable to that of acoustic source stimulation. However, at low frequency range, for all the temporal bones tested, the magnitude of stapes velocity caused by the round window stimulation was lower than tympanic membrane stimulation. Thus, more driving current is needed to produce $94 \mathrm{~dB}$ SPL in the low frequency region.

The magnitude of the stapes velocity by the round window stimulation at $1 \mathrm{~mA}_{\mathrm{rms}}$ was equivalent to that of stapes velocity at $94 \mathrm{~dB}$ SPL stimulation. Therefore, the 3-pole type EM transducer can stimulate the cochlea to the same degree as tympanic membrane stimulation. Accordingly, it is expected that the 3-pole type EM transducer can be used in implantable hearing aids. Further studies are required to optimize the coupling between the actuator and the round window.

\section{Acknowledgement}

This study was supported by a grant from the Korea Healthcare Technology R \& D Project, Ministry of Health \& Welfare (No. A092106), a National Research Foundation of Korea (NRF) grant funded by the Korea government (MSIP) (No. 2013R1A2A1A09015677), and the Basic Science Research Program through the NRF funded by the Ministry of Education (No. 2013R1A1A2004991).

\section{References}

[1] R. Goode, M. Rosenbaum and A. Maniglia, The history and development of the implantable hearing aid, The Otolaryngologic Clinics of North America 28 (1995), 1-6.

[2] B.S. Wilson and M.F. Dorman, Cochlear implants: A remakable past and a brilliant future, Hearing Research 242 (1995), 3-21.

[3] H. Dillon, Hearing Aids, Boomerang Press, Sydney, Australia, 2001, pp. 1-16.

[4] H. H. Kim and D.M. Barrs, Hearing aids: A review of what's new, Journal of Otolaryngology-Head and Neck Surgery, 134 (2006), 1043-1050.

[5] J. Suzuki, H. Shono, K. Koga and T. Akiyama, Early studies and the history of development of the middle ear implant in Japan, Advances in Audiology 4 (1988), 1-14.

[6] A. Chasin, Current trends in implantable hearing aids, Trends in Amplification 2 (1997), 84-107.

[7] S.E. Voss, J.J. Rosowski and W.T. Peake, Is the pressure difference between the oval and round window the effective acoustic stimulus for the cochlea? Journal of the Acoustical Society of America 100 (1996), 1602-1616.

[8] H.H. Nakajima, W. Dong, M.E. Ravicz, S.N. Merchant, E.S. Olson and J.J. Rosowski, Evaluation of round window stimulation using the floating mass transducer by intracochlear sound pressure measurements in human temporal bones, Otol. Neurotol. 31 (2010), 506-511.

[9] A. Arnold, M. Kompis, C. Candreia, F. Pfiffner, R. Hausler and C. Stieger, The floating mass transducer at the round window: Direct transmission or bone conduction? Hearing Research 263 (2010), 120-127. 
[10] Y. Shimizu, S. Puria and R. Goode, The floating mass transducer on the round window versus attachment to an ossicular replacement prosthesis, Otol. Neurotol. 32 (2011), 98-103.

[11] D.H. Shin, D.W. Kim, H.G. Lim, E.S. Jung, K.W. Seong, J.H. Lee, M.N. Kim and J.H. Cho, Measurement of stapes vibration in human temporal bones by round window stimulation using a 3-coil transducer, Bio-Medical Materials and Engineering 24 (2014), 405-411.

[12] J.H. Spindel, P.R. Lambert and R.A. Ruth, The round window electromagnetic implantable hearing aid approach, Otolaryngol Clinics of North America 28 (1995), 189-205.

[13] V. Colletti, S.D. Soli, M. Carner and L. Colletti, Treatment of mixed hearing losses via implantation of a vibratory transducer on the round window, International Journal of Audiology 45 (2006), 600-608.

[14] K.W. Seong, M.W. Kim, J.H. Lee, M.N. Kim and J.H. Cho, Design of a new vibration transducer for implantable middle ear hearing devices, Institute of Electrical Engineers of Japan 5 (2010), 608-610.

[15] ASTM International, Standard Practice for Describing System Output of Implantable Middle Ear Hearing Devices, Designation: F2504-05.

[16] T.G. Dietz, G.R. Ball and B.H. Katz, Partially implantable vibrating ossicular prosthesis, International Conference on Solid-State Sensors and Actuator 1 (1997), 433-436.

[17] D.W. Kim, K.W. Seong, M.N. Kim and J.H. Cho, A 1-channel 3-band wide dynamic range compression chip for vibration transducer of implantable hearing aids, Bio-medical materials and engineering 24 (2014), 1009-1017. 